\title{
Seguimento de crianças com desnutrição moderada ou grave em população periférica (Brasil)*
}

\author{
Follow up of children with moderate or severe malnutrition in an outlying \\ urban population (Brazil)
}

\author{
Nilson M. Carvalho**, Elsa R.J. Giugliani**, Cristina F. Seffrin ${ }^{* *}$, Rosane M. Hartmann ${ }^{\star *}$
}

\begin{abstract}
CARVALHO, N.M. et al . Seguimento de crianças com desnutricão moderada ou grave em população periférica (Brasil). Rev. Saúde públ., S.Paulo, 26: 223-8, 1992. Com o objetivo de avaliar o estado nutricional de crianças de uma população urbana periférica de Porto Alegre, RS (Brasil) que apresentaram quadro de desnutriçăo moderada ou grave antes dos 5 anos de idade, 61 famílias foram procuradas após 2 a 4 anos de uma avaliação inicial. Das 39 crianças localizadas, $4(10,3 \%)$ foram a óbito e $22(56,4 \%)$ apresentaram um incremento na relação peso/idade maior que $10 \%$. Entre as 35 crianças sobreviventes, $29(82,3 \%)$ apresentavam algum grau de desnutrição (peso/idade $\leq 90 \%$ do padrão), 25 (71,4\%) tinham baixa estatura (altura/idade $\leq 95 \%$ do padrão) e $5(14,3 \%$ ) possuíam pouco peso para a altura $\leq 90 \%$ do padrão). Os irmãos menores de 5 anos de idade apresentaram estado nutricional semelhante ao das crianças reavaliadas. Os fatores que mostraram alguma associação com um melhor estado nutricional (incremento maior que $10 \%$ na relação peso/idade no periodo do seguimento e/ou altura/idade ou peso/altura adequados na segunda avaliacão) foram: história de pelo menos uma hospitalização entre a primeira e a segunda avaliação, detecção da desnutrição até os 6 meses de idade e mãe alfabetizada. Os programas de suplementação alimentar e/ou reabilitação nutricional disponiveis na comunidade não influíram na melhoria do estado nutricional, tanto das crianças-alvo como de seus irmãos. Concluiu-se pela necessidade de uma abordagem mais eficaz das famílias que apresentam um alto risco para desnutrição e morbimortalidade infantil.
\end{abstract}

Descritores: Desnutrição protéico-energética, epidemiologia. Suplementação alimentar. Estado nutricional.

\section{Introdução}

A desnutrição infantil é um problema mundial de saúde pública. Estima-se que no mundo inteiro cerca de 100 milhōes de crianças sofram de desnutrição moderada ou gra$\mathrm{ve}^{1}$. Em algumas regiōes do Brasil mais da metade das crianças é desnutrida ${ }^{8}$, sendo que muitas morrem pela própria desnutriçāo ou por doenças a ela associadas, principalmente infeç̧ões.

Com freqüência o profissional de saúde detecta a desnutrição, por vezes grave, mas, sozinho, pouco pode fazer pelo seu paciente, devido à multiplicidade de fatores que contribuem para o estabelecimento e a manutenção do quadro ${ }^{5}$. O manejo da desnutrição, portanto, pode ser complexo, envolvendo profissio-

* Pesquisa subvencionada pelo Conselho Nacional de Desenvolvimento Científico e Tecnológico (Processo $\mathrm{n}^{2}$ 408970-87.1).

* Departamento de Pediatria e Puericultura da Faculdade de Medicina da Universidade Federal do Rio Grande do Sul - Porto Alegre, RS - Brasil.

Separatas/Reprints: E.R.J. Giugliani - Hospital das Clinicas de Porto Alegre - Rua Ramiro Barcelos, 2350 - 90035-003

Porto Alegre, RS - Brasil. nais de diversas áreas e, em muitos casos, exigindo um programa de suplementação alimentar para reabilitar nutricionalmente a criança desnutrida.

Existem inúmeros programas de suplementação alimentar na América Latina e no Caribe $^{3}$, a maioria financiada pelos governos e atendendo a pré-escolares. Nas últimas décadas foram criados vários desses programas no Brasil. No entanto, apesar da falta de avaliação do impacto desses programas no estado nutricional da populacão-alvo, é sabido que a maioria deles é insuficiente tanto na extensão de cobertura como no aporte nutricional fornecido ${ }^{10}$.

Nos anos de 1984 e 1985 foi realizado estudo sobre fatores de alto risco para desnutrição moderada ou grave no primeiro ano de vida numa população urbana periférica (Porto Alegre, RS) ${ }^{4,5}$. As crianças incluídas nesse estudo e outras avaliadas no mesmo período e que se desnutriram nos primeiros 5 anos de vida foram encaminhadas para participarem de programas de reabilitação nutricional e/ou suplementação alimentar existentes na comunidade. Após 2 a 4 anos essas crianças foram 
reavaliadas com os seguintes objetivos:

1. Avaliar o estado nutricional de crianças que apresentaram desnutrição moderada ou grave antes dos 5 anos de idade, após 2 a 4 anos de evolução;

2. Avaliar o estado nutricional dos irmãos menores de 5 anos dessas crianças;

3. Verificar a influência dos programas de apoio nutricional disponíveis na comunidade na melhoria do estado nutricional dessas crianças;

4. Identificar fatores que interferiram na evolucão do estado nutricional das ctianças no periodo estudado.

\section{Material e Métodos}

Um grupo de 61 mães ou substitutas de crianças com desnutrição moderada (relação peso/idade entre 61 e $75 \%$ ) ou grave (relação peso/idade $\leq 60 \%$ ), de acordo com os padrões estabelecidos por Marques e col. ${ }^{9}$, foi entrevistado nos anos de 1984 e 1985, com o objetivo de identificar fatores de alto risco para desnutrição moderada ou grave numa população urbana periférica.

As crianças, todas menores de 5 anos na época, foram selecionadas aleatoriamente na Unidade Sanitária Vila Cruzeiro do Sul, localizada em Porto Alegre, RS. Deste grupo inicial de 61 crianças, $46(75,4 \%)$ apresentavam desnutrição moderada e $15(24,6 \%)$ desnutrição grave.

Transcorridos no mínimo 22 e no máximo 52 meses da primeira entrevista, tentou-se localizar o grupo de crianças desnutridas para reavaliação. As 39 mães ou substitutas $(63,9 \%)$, que puderam ser localizadas, foram entrevistadas utilizando-se questionário padronizado contendo perguntas a respeito da participação das crianças em programas de apoio nutricional, além de questões que visavam à pesquisa de fatores que pudessem interferir na evolução do estado nutricional do grupo em estudo.

As crianças desnutridas que foram localizadas e seus irmãos menores de 5 anos foram pesados com balança de haste portátil marca Cauduro, e medidas com antropômetro de madeira (crianças até 2 anos de idade) ou fita métrica, quanto ao seu comprimento ou à sua altura respectivamente. As relações peso/idade e altura (ou comprimento)/idade foram calculadas utilizando-se como referência a tabcla nivel IV de Marques e col. ${ }^{9}$. Para fins de comparação com outros trabalhos realizados na mesma área do presente estudo, utilizou-se a classificação de desnutrição proposta por Waterlow $^{16}$, que considera baixas as crianças com altura (ou comprimento) igual ou menor que 95\% do padrão para a idade e leves as com peso igual ou menor que $90 \%$ do padrão para a altura. A classifícação socioeconômica utilizada foi a de Graciano ${ }^{7}$, que leva em consideração o número de membros da família, a escolaridade e a profissão dos pais, o tipo de habitação e a renda familiar.

Para a análise estatística foi utilizado o teste do qui-quadrado, aceitando-se como significante um $\mathrm{p}<0.05$.

\section{Resultado}

Das 61 mães ou substitutas que foram entrevistadas inicialmente, $22(36,1 \%)$ não puderam ser localizadas. Segundo informações de vizinhos, 6 dessas famílias teriam se mudado para o interior do Estado. Dentre as famílias encontradas, $15(38,5 \%)$ haviam mudado de domicílio pelo menos uma vez. Comparando o grupo que foi submetido à segunda avaliação com o grupo que não foi localizado, não houve diferença estatisticamente significante quanto ao sexo, à idade, ao grau de desnutrição, à classificação socioeconômica das crianças e à idade na detecção da desnutrição.

Das 39 crianças cuja evolução foi conhecida, $4(10.3 \%)$ foram a óbitos. Infelizmente as famílias haviam extraviado os atestados de óbitos e não sabiam informar a causa imediata da morte. Porém houve o relato de meningite tuberculosa em 2 desses casos.
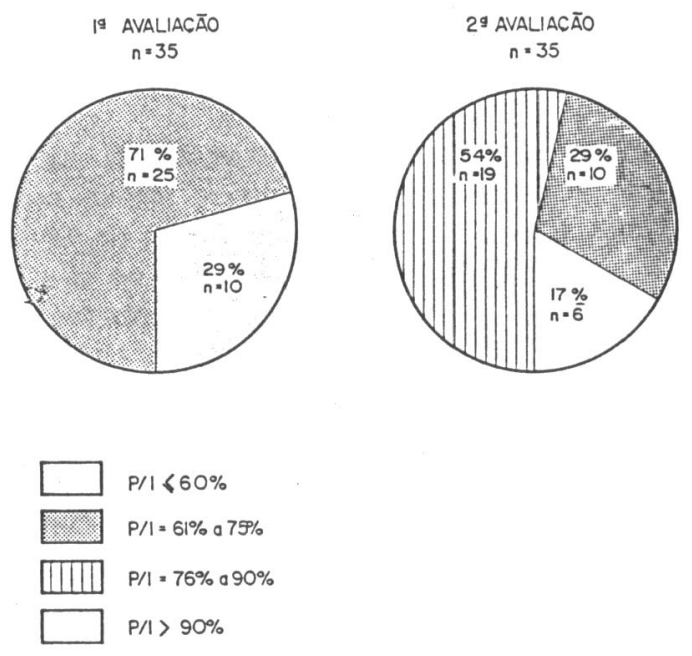

Figura 1. Peso/idade das crianças na primeira e na segunda avaliação. 
A idade média das 35 crianças (18 meninas e 17 meninos) sobreviventes na segunda avaliação foi de $67,4 \pm 18,4$ meses. A Figura 1 apresenta $o$ estado nutricional das crianças estudadas, na primeira e na segunda avaliação, segundo o peso para a idade. Nenhuma criança apresentou desnutrição grave na segunda avaliação e $6(17,1 \%)$ passaram a ter peso adequado para a idade. A Figura 2 mostra as diferenças na relação peso/idade das crianças entre a primeira e a segunda avaliação. A maioria $(62,9 \%)$ teve um incremento maior que $10 \%$ na relação peso/idade e uma minoria $(14,3 \%)$ teve a relação diminuída.

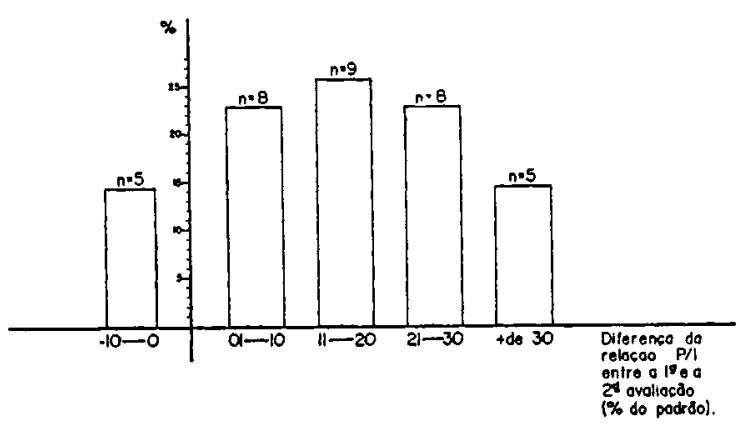

Figura 2. Incremento na relação peso/idade das crianças entre a primeira e a segunda avaliação

Aproximadamente metade das crianças reavaliadas participou de programas de suplementação alimentar. Considerando como incremento segnificativo de peso uma variação na relação peso/idade maior que 10 pontos percentuais, as crianças que participaram de algum programa de apoio nutricional não diferiram das demais quanto ao incremento de peso no periodo estudado (Tabela 1).

Tabela 1. Incremento na relaçăo peso/idade das crianças no período estudado, de acordo com a participa. ção ou nāo em programas de apoio nutricional.

\begin{tabular}{|c|c|c|c|c|}
\hline \multirow{3}{*}{$\begin{array}{l}\text { Participação em } \\
\text { programas de apoio } \\
\text { nutricional }\end{array}$} & \multicolumn{4}{|c|}{ Incremento na relacão peso/idade } \\
\hline & & $>10 \%$ & & $\leq 10 \%$ \\
\hline & $n$ & $(\%)$ & $n$ & $(\%)$ \\
\hline $\operatorname{sim}$ & 12 & $(70,6)$ & 5 & $(29,4)$ \\
\hline não & 10 & $(55,6)$ & 8 & $(44,4)$ \\
\hline
\end{tabular}

$X 2=0,85 \quad p>0,05$

Pouco mais da metade das famílias estudadas $(57,9 \%)$ manteve a mesma classificação socioeconômica entre a primeira e a segunda avaliação. Praticamente $1 / 4(21 \%)$ das crianças melhorou de nível socioeconômico segun- do a classificação utilizada e o restante $(21 \%)$ passou a um nível inferior. A mudança da classificação socioeconômica não influiu no incremento de peso das crianças desnutridas (Tabela 2).

Tabela 2. Incremento na relação peso/idade das crian. ças no periodo estudado, de acordo com a mudança do nivel socioeconômico familiar.

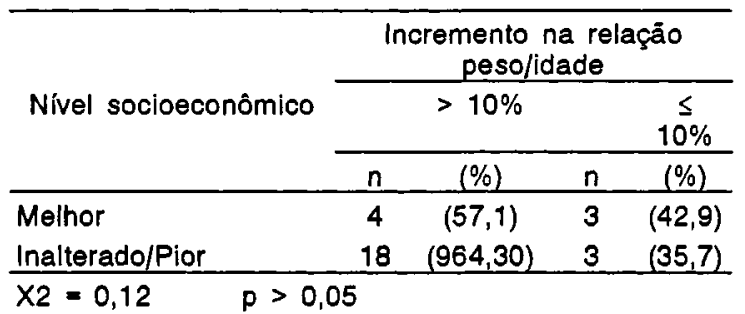

Cerca de metade das crianças avaliadas $(51,4 \%)$ teve pelo menos uma hospitalização no período do seguimento. As crianças hospitalizadas tiveram um incremento de peso significantemente maior que as não hospitalizadas (Tabela 3).

Tabela 3. Incremento na relação peso/idade das criancas no período estudado, de acordo com a história de hospitalização nesse periodo.

\begin{tabular}{|c|c|c|c|c|}
\hline \multirow{3}{*}{$\begin{array}{l}\text { História de } \\
\text { hospitalização }\end{array}$} & \multicolumn{4}{|c|}{ Incremento na relacão peso/idade } \\
\hline & \multicolumn{3}{|c|}{$>10 \%$} & \multirow{2}{*}{$\frac{\leq 10 \%}{(\%)}$} \\
\hline & $\underline{n}$ & $(\%)$ & n & \\
\hline $\operatorname{sim}$ & 15 & $(83,3)$ & 3 & $(16,7)$ \\
\hline năo & 7 & $(41,2)$ & 10 & $(58,8)$ \\
\hline
\end{tabular}

Quando comparadas as crianças cuja desnutrição foi detectada os primeiros 6 meses de idade com as demais observou-se que as primeiras apresentaram um incremento de peso significativamente maior (Tabela 4).

Tabela 4. Incremento na relação peso/idade das crianças no periodo estudado, de acordo com a idade na detecção da desnutrição.

\begin{tabular}{lcccc}
\hline & \multicolumn{3}{l}{ Incremento na relação peso/idade } \\
\cline { 2 - 5 } $\begin{array}{l}\text { ldade na detecção } \\
\text { da desnutrição }\end{array}$ & \multicolumn{4}{c}{$>10 \%$} \\
& $n$ & $(\%)$ & $n$ & $(\%)$ \\
\cline { 2 - 5 } & 11 & $(91,7)$ & 1 & $(8,3)$ \\
$\leq 6$ meses & 11 & $(47,8)$ & 12 & $(52,2)$ \\
\hline 6 meses & $p<0,05$ & & &
\end{tabular}

A Figura 3 mostra as relações altura/idade e peso/altura das crianças na segunda avalia- 


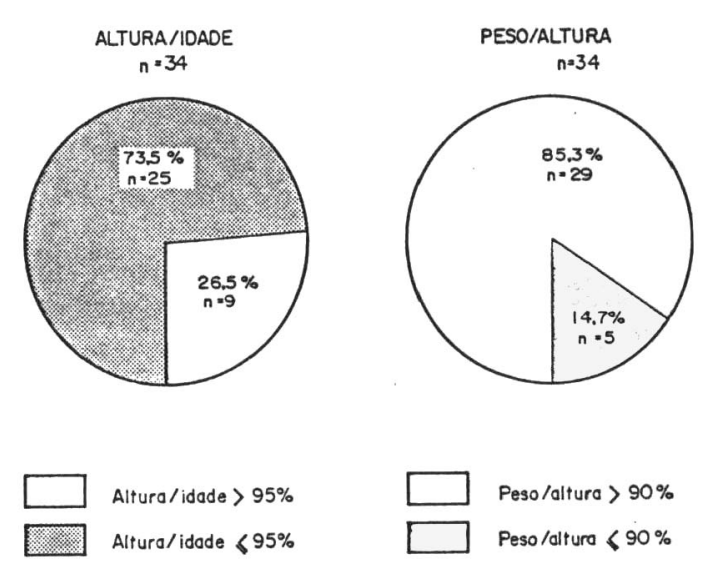

Figura 3. Altura/idade e peso/altura das crianças na segunda avaliação.

ção. Infelizmente não foi possível a comparação com os índices anteriores por não se dispor da altura das crianças na época da primeira entrevista. A Tabela 5 apresenta as associações existentes entre baixa estatura (altura/idade $\leq 95 \%$ ) e peso inadequado para a altura (peso/altura $\leq$ $90 \%) \mathrm{com}$ algumas variáveis estudadas. As crianças cuja desnutrição foi detectada precocemente (até os 6 meses) possuíam, na segunda avaliação, uma altura mais adequada para a idade. Por outro lado, aquelas que tiveram pelo menos uma hospitalização ou cujas mães ou substitutas eram alfabetizadas apresentaram uma relação peso/altura mais adequada.

Tabela 5. Associação entre baixa estatura (altura/idade $\leq 95 \%$ ) e peso insuficiente para a altura (peso/altura $\leq 90 \%$ ) das crianças e algumas variáveis estudadas.

\begin{tabular}{lll}
\hline Variáveis & \multicolumn{2}{c}{ Qui-quadrado } \\
\cline { 2 - 3 } & Al! & P/A \\
\hline $\begin{array}{l}\text { Participação em programa de } \\
\text { apoio nutricional }\end{array}$ & 0,93 & 0,88 \\
$\begin{array}{l}\text { Idade na detecção da desnutrição } \\
\text { (> } 6 \text { meses, } \leq 6 \text { meses) }\end{array}$ & $5,27 *$ & 1,25 \\
$\begin{array}{l}\text { Hospitalização entre a primeira e } \\
\text { a segunda avaliação (sim, nāo) }\end{array}$ & 0,15 & $5,20 *$ \\
$\begin{array}{l}\text { Escolaridade materna } \\
\text { (alfabetizada, nāo alfabetizada) }\end{array}$ & 1,05 & $4,33^{*}$ \\
\hline $\begin{array}{l}\text { Al - altura/idade P/A - peso/altura } \\
\text { * estatisticamente significante }\end{array}$ & &
\end{tabular}

Das 39 crianças que foram reavaliadas, 28 tinham irmãos menores de 5 anos, perfazendo um total de 39 irmãos. A Tabela 6 mostra o resultado das avaliações antropométricas realizadas nessas crianças. Cerca de $80 \%$ delas apresentavam algum grau de desnutrição
Tabela 6. Estado nutricional dos irmãos menores de 5 anos das crianças estudadas

\begin{tabular}{ccc}
\hline Parâmetro & $n$ & $(\%)$ \\
\hline Peso/idade & & \\
$>90 \%$ & 8 & $(20,5)$ \\
76 a $90 \%$ & 23 & $(59,0)$ \\
$\leq 75 \%$ & 8 & $(20,5)$ \\
& & \\
Altura/idade & & \\
$>95 \%$ & 13 & $(33,3)$ \\
$\leq 95 \%$ & 26 & $(66,7)$ \\
Peso/altura & & \\
$>90 \%$ & & \\
$\leq 90 \%$ & 30 & $(66,9)$ \\
& 9 & $(23,1)$ \\
\hline
\end{tabular}

segundo o peso para a idade, $2 / 3$ tinham baixa estatura, porém a maioria $(76,9 \%)$ tinha um peso adequado para a altura. Os irmãos das crianças que participaram de programas de apoio nutricional não apresentaram melhor estado nutricional que os demais (Tabela 7).

Tabela 7. Estado nutricional dos irmäos menores de 5 anos, das crianças estudadas, de acordo com a par. ticipaçăo das crianças desnutridas em programas de apoio nutricional.

\begin{tabular}{|c|c|c|c|c|c|}
\hline \multirow{3}{*}{ Parâmetro } & \multicolumn{4}{|c|}{$\begin{array}{c}\text { Participação dos } \\
\text { irmäos desnutridos em } \\
\text { programas de apoio } \\
\text { nutricional }\end{array}$} & \multirow{3}{*}{ Qui-quadrado } \\
\hline & \multicolumn{3}{|c|}{ sim } & \multirow{2}{*}{$\frac{\text { não }}{(\%)}$} & \\
\hline & $n$ & $(\%)$ & $n$ & & \\
\hline Peso/idade & & & & & 0,06 \\
\hline$>90 \%$ & 4 & $(50,0)$ & 4 & $(50,0)$ & \\
\hline$\leq 90 \%$ & 14 & $(45,2)$ & 7 & $(54,8)$ & \\
\hline Altura/idade & & & & & 0,46 \\
\hline$>95 \%$ & 5 & $(38,5)$ & 8 & $(61,5)$ & \\
\hline$\leq 95 \%$ & 13 & $(50,0)$ & 13 & $(50,0)$ & \\
\hline Peso/altura & & & & & 0,42 \\
\hline$>90 \%$ & 13 & $(43,3)$ & 17 & $(56,7)$ & \\
\hline$\leq 90 \%$ & 5 & $(55,6)$ & 4 & $(44,4)$ & \\
\hline
\end{tabular}

\section{Discussão}

É bem conhecido o fato de que crianças desnutridas apresentam uma maior morbimortalidade $^{2}$. Uma pesquisa realizada pela Organização Panamericana de Saúde, em 12 cidades das Américas mostrou que a desnutrição, embora nem sempre registrada nos atestados de óbito, é a principal causa básica ou associada de mortalidade infantil em populações 
de baixa renda ${ }^{11}$. $O$ mesmo estudo apontou a desnutrição da criança ou da mãe como a principal responsável pela mortalidade infantil em 3 cidades brasileiras. Os resultados do presente trabalho vieram corroborar com os dados já existentes na literatura. A mortalidade no grupo estudado foi de $103 / 1000$, taxas 5 vezes maior que a observada para crianças de 1 a 5 anos no Brasil $(24 / 1000)^{14}$. Paralelamente à alta mortalidade foi observada uma elevada morbidade, visto que metade das crianças foi hospitalizada pelo menos uma vez entre a detecção da desnutrição e a avaliação posterior. Fato curioso é que aquelas crianças com história de hospitalização apresentaram melhor padrão de seu estado nutricional na segunda avaliação, tanto no que se refere ao incremento na relação peso/idade, no período avaliado, quanto ao peso para altura. Este achado reflete a desordem existente em sistema de saúde do país com um serviço de atendimento primário ineficiente para superar os determinantes econômicos, sociais e culturais da desnutrição infantil, e os já saturados serviços secundários e terciários assumindo um papel que não lhes compete. O custo social desta distorção é enorme, e a solução certamente envolve uma série de questões que abrange a própria organização politica e econômica da sociedade brasileira. Um agravante desta situação é a freqüente mudança de domicílio encontrada em populações urbanas periféricas, fato confirmado pelo presente estudo. As famílias, muitas vezes, não chegam a formar um vínculo com o posto de sáude e, com freqüência, devido à mudança de domicílio, interrompem a sua participação em programas de saúde, como por exemplo em programas de reabilitação nutricional.

Apesar da maioria das crianças sobreviventes apresentar uma melhora no estado nutricional no periodo de acompanhamento, 0 número de crianças que ainda apresentavam algum grau de desnutrição é bastante elevado (83\% da amostra). Igualmente elevado foi o índice de desnutrição encontrado entre os irmãos menores de 5 anos quando comparamos com os dados da Pesquisa Nacional sobre Saúde e Nutrição ${ }^{8}$, realizada em todo o país, no ano de 1989. Esta pesquisa revelou uma prevalência de desnutrição em crianças menores de 5 anos igual a $30,7 \%$, com $5,1 \%$ de crianças com desnutrição moderada ou grave. O contraste é ainda maior se for levado em consideração apenas a região onde foi realizado o estudo (região sul), com uma preva- lência de desnutrição infantil de $17,8 \%$.

Cerca de $75 \%$ das crianças estudadas e 67\% dos irmãos apresentaram baixa estatura enquanto que aproximadamente $15 \%$ dos propósitos e $23 \%$ dos irmãos tinham peso insuficiente para altura. Em pesquisa realizada por Giugliani e col. ${ }^{6} \mathrm{em}$ uma área bem definida, na mesma localidade onde foi realizado o presente estudo, a prevalência de crianças menores de 5 anos de idade com baixa estatura foi de $44,3 \%$ e com peso insuficiente para a altura de $9,5 \%$. Quando se analisa a altura e o peso para a altura no grupo de crianças com desnutrição moderada ou grave prévia pode-se concluir que a maioria dessas crianças apresenta atualmente caráter crônico de desnutrição, atingindo um certo equilíbrio entre o peso e a estatura. Os irmãos menores de 5 anos apresentaram um padrão nutricional semelhante. Estas crianças, uma vez adultos, terão uma estatura mais baixa do que aquelas que nunca desnutriram e certamente estatão em desvantagem, já que a produtividade e a capacidade de trabalho físico estão relacionados com o tamanho corporal ${ }^{13}$.

Cerca de metade das crianças estudadas participou de programas oficiais de apoio e reabilitação nutricionais, o que não alterou a evolução de seu estado nutricional. Tais programas se baseiam quase que exclusivamente na distribuição de leite e de outros alimentos às famílias, $o$ que explica em parte o fracasso de seus resultados. Nas duas últimas décadas foram criados vários programas de nutrição infantil no Brasil, porém na maioria dos casos os resultados ficaram aquém do esperado ${ }^{10,12}$. As explicações para os insucessos são várias, incluindo desde falhas administrativas, inadequação no estabelecimento de metas deficiências orçamentárias, até interesses político-eleitoreiros, muitas vezes subjacentes. $\mathrm{Na}$ realidade, esses programas, mesmo funcionando eficientemente, apenas conseguem contribuir para um alívio da situação, já que as soluções para o problema da desnutrição infantil incluem mudanças de ordem política e social.

Mais importante que a participação em programas de apoio nutricional para um incremento significativo de peso foi a idade da detecção da desnutrição. Aquelas crianças cuja desnutrição foi detectada nos primeiros $6 \mathrm{me}$ ses de vida não só tiveram um incremento maior de peso no período estudado como apresentaram uma altura mais adequada para a idade na reavaliação. Daí a importância do seguimento das crianças desde o seu nascimento. 
No presente estudo as crianças cujas mães são alfabetizadas apresentaram uma relação peso/altura mais adequada, o que vem reforçar os dados da literatura que mostram a importância da educaçāo materna no estado nutricional de seus filhos ${ }^{15}$.

Pode-se concluir que as familias abordadas no presente estudo apresentam um perfil social, econômico, cultural e biológico que as colocam em alto risco para desnutrição e morbimortalidade infantil. Os programas oficiais de apoio nutricional a estas famílias pouco tem influenciado na melhoria do estado nutricional tanto das crianças-alvo como de seus irmãos, exigindo uma abordagem mais eficaz.

CARVALHO, N.M. et al. [Follow up of children with moderate or severe malnutrition an outlying urban population (Brazil)]. Rev. Saúde públ., S.Paulo, 26: 223-8, 1992. What happens to children who develop moderate or severe malnutrition? What is done for them? Keeping in mind these questions, the present research was undertaken with the following objectives: to assess the nutritional status of children who develop moderate or severe malnutrition before the age of 5 years, after a period from 2 to 4 years after diagnosis; to assess the nutritional status of the under 5-year old siblings of these children; to study the influence of nutritional programs available in the community for the improvement of the nutritional status of the malnourished children; and to identify factors interfering with nutrition of these children during the study period. After a period of 2 to 4 years from the time of diagnosis of moderate or severe malnutrition the authors tried to locate the families of 61 malnourished children of Porto Alegre, RS (Brazil). The mothers their substitutes were interviewed and the children and siblings under 5 years of age were weighed and measured. Thirty-nine children were located. Of these, $4(10 \%)$ died and $22(56 \%)$ presented an increase of at least $10 \%$ in weight for age. Of the 35 children who survived, $29(82 \%)$ still presented some degree of malnutrition (weight/age $\leq 90 \%$ of the standard), $25(71 \%)$ were stunted (height/age $\leq$ $95 \%$ ), and $5(14 \%)$ were wasted (weight/height $\leq$ $90 \%)$. The nutritional status of the 5 -year old siblings was similar to that of the malnourished children. The factors showing some association with better nutrition (an increase in weight/age of $10 \%$ or more during the study period and/or adequate height/age or weight/height) were: history of at least one hospitalization during follow-up, early detection of malnutrition (before the age of 6 months), and literate mothers. The nutritional programs available in the community did not show any influence on the nutritional status of the malnourished children and their siblings. The present study shows that a more efficient intervention is necessary in the families who are at higher risk for infant morbimortality.

Keywords: Protein-caloire malnutrition, epidimiology. Supplementary feeding. Nutritional status

\section{Referências Bibliográficas}

1. EBRAHIM, G.J. Protein-energy malnutrition. In: Ebrahim, G.J. Nutrition in mother and child health. London, Macmillan, 1983. p. 104-33.

2. EBRAHIM, G.J. Pattems of morbidity and mortality. In: Ebrahim, G.J. Social \& community paediatrics in developing countries. London, Macmillan, 1985. p. 77-95.

3. ESPINOSA, E.; RUIZ, C.; VALENTE, S. Programas de intervencion alimentario-nutricional en America Latina y el Caribe entre 1970-1984. Food Nutr. Bull., 8:17-23, 1986.

4. GIUGLIANI, E.R.J.; SEFFRIN, C.F.; GOLDANI, M.; HORN, J.F.C.M.; EBRAHIM, G.J. The malnourished children of the urban squatter families: a study in Porto Alegre, Brazil. J. trop. Pediat., 33:194-8, 1987.

5. GIUGLIANI, E.R.J.; SEFFRIN, C.F.; GOLDANI, M., HORN, J.F.C.M. Fatores de alto risco para desnutrição em populações urbanas periféricas. $J$. Pediat., 65: 114-8, 1989.

6. GUIGLIANI, E.RJ.; ROTTA, A.T.; RIBEIRO, A.M.; MELLO, C.; MOREIRA, C.M.; DIAS, C.C.C.; PRYTALUK, T.M. Percepção materna sobre a adequação do peso e da altura de crianças menores de 5 anos em uma vila periférica de Porto Alegre. Rev. HCPA, 10:70-3, 1990.

7. GRACIANO, M.I.G. Critérios de avaliação para classificaçảo sócio-econômica. Serv. soc. e Soc., 8: 81-103, 1980.

8. INSTITUTO NACIONAL DE ALIMENTAÇĀO E NUTRIÇÃO. Pesquisa nacional sobre saúde $e$ nutrição: perfil de crescimento da população brasileira de 0 a 25 anos. Brasília, 1990.

9. MARQUES, RM.; BERQUÓ, E.; YUNES, J.; MARCONDES, E. Crescimento de crianças brasileiras: peso e altura segundo idade e sexo. Influência de fatores sócio-econômicos. An. Nestlé, (84 supl. 2) 1974.

10. PELIANO, A.M. et al. Considerações sobre os programas govemamentais de suplementaçāo alimentar. Fome Deb., Brasília, 7: 13, 1989.

11. PUFFER, R.R. \& SERRANO, C.V. Patterns of mortality in childhood. Washington, D.C., Pan American Health Organization, 1973.

12. SILVA, A.C. Desnutrição: Nordeste, desespero ou esperança. Ciênc. Hoje, 1: 64-70, 1983.

13. SPURR, G.B. Tamaño corporal, capacidad de realizar trabajos fisicos y productividade en el trabajo intenso: es mejor mas grande? In: Retraso del crecimiento linel en los paises en vias de desarrollo. New York, Ravem Press, 1987. p. 25-9.

14. UNICEF. The state of the world's children. Oxford, Oxford University Press, 1991.

15. VARGAS, LA. Contexto socioantropologico del crecimento infantil. In: Cusminsky, M.; Moreno, E.M., Suarez Ojeda, E.N. Crecimento y desarrollo: hechos y tendencias. Washington, D.C., Organizacion Panamericana de la Salnd, 1988. (OPAS -Publicacion Cientifica 510).

16. WATERLOW, J. Some aspects of childhood malnutrition as a public health problem. Brit. med. J., 4: 88-90, 1974. 